\section{Axiale Spondyloarthritis: Langzeiteffekt auf die körperliche Aktivität in der Freizeit}

\author{
Sveaas SH et al. Longterm Effect on Leisure Time \\ Physical Activity Level in Individuals with Axial \\ Spondyloarthritis: Secondary Analysis of a \\ Randomized Controlled Trial. J Rheumatol 2020; \\ 47: 1189-1197. doi:10.3899/jrheum.190317
}

Die axiale Spondyloarthritis betrifft vorwiegend das Achsenskelett. Die Erkrankung ist gekennzeichnet durch entzündlich bedingten Rückenschmerz und kann auch zu einer verminderten Beweglichkeit der Wirbelsäule, zu Arthritis, Enthesitis, Steifheit und Fatigue führen. Zudem ist das Risiko, eine kardiovaskuläre Erkrankung zu entwickeln, erhöht.

Körperliche Aktivität wird als ein wichtiger Bestandteil des Managements bei axialer Spondyloarthritis angesehen, aber es gibt die Befürchtung, dass zu intensives Training schädlich sein und die Erkrankung zur Exazerbation bringen könnte. Inzwischen wird empfohlen, entweder $5 \mathrm{Mal}$ pro Woche über mindestens $30 \mathrm{Min}$ ein leichteres, oder 3 Mal pro Woche für mindestens 20 Min ein intensiveres Training durchzuführen, ergänzt durch ein Krafttraining 2-3 Mal pro
Woche. Dies zeigt eine Entwicklung hin zu mehr kardiorespiratorischen Übungen und Krafttraining.

\section{Ziel}

In dieser Studie wird die Langzeitwirkung eines dreimonatigen Trainingsprogramms auf die körperliche Aktivität in der Freizeit bei Personen mit axialer Spondyloarthritis (axSpA) untersucht.

\section{Methoden}

Die Studienautoren führten eine Sekundäranalyse von Daten über 100 Personen mit axSpA durch und schlossen diese in eine randomisierte kontrollierte Studie ein. Die „Übungsgruppe“ nahm an einem 3-monatigen Übungsprogramm teil, während die „Kontrollgruppe“ keine Intervention erhielt. Die körperliche Aktivität in der Freizeit maßen die Untersucher mit einem Fragebogen (körperlich aktiv: $\geq 1$ h/Woche bei mäßiger/starker körperlicher Aktivität). Die Krankheitsaktivität bestimmten sie mithilfe der Aktivitätsskala für die ankylosierende Spondylitis (ASDAS; höhere Punktzahl = schlechteste). Die Wissenschaftler führten statistische Analysen auf Basis von Behandlungsabsichten unter Verwendung von ChiQuadrat-Tests, logistischer Regression und gemischten Modellen durch.

\section{Ergebnisse}

Bei der 12-monatigen Nachuntersuchung waren signifikant mehr Personen in der Übungsgruppe als in der Kontrollgruppe körperlich aktiv (29 [67\%] gegenüber 13 [30\%], $p<0,001)$. Sie trainierten 2-3 Mal pro Woche (25 [58\%]) vs. 15 [34\%]; $p=0,02)$ und weniger bei Lichtintensität (3 [8\%] vs. 14 [44\%]; $p=0,002)$. „Teilnahme an der Übungsgruppe“ (OR: 6,7; 95\%-KI: 2,4-18,6; $\mathrm{p}<0,001)$ und ,körperliche Aktivität zu Studienbeginn" (OR: 4,7; $95 \%$-KI: 1,4-15,8; $\mathrm{p}=0,01)$ waren die mit körperlicher Aktivität am häufigsten assoziierten Faktoren. Bezüglich der ASDAS gab es keine Unterschiede zwischen den Gruppen ( $p=0,79)$.

\section{FAZIT}

Bei Patienten mit axialer Spondyloarthritis wirkt sich ein 3-monatiges Trainingsprogramm langfristig positiv auf die körperliche Aktivität in der Freizeit aus, was auf ein verbessertes Gesundheitsprofil hinweist. Dennoch setzten nur wenige Personen das Intensivprogramm fort, und nach 12 Monaten gab es in Bezug auf die Krankheitsaktivität keinen Unterschied zwischen den Gruppen.

Dr. Catharina Brandes, Gmund am Tegernsee 